TRANSACTIONS OF THE

AMERICAN MATHEMATICAL SOCIETY

Volume 194, 1974

\title{
TRANSVERSALLY PARALLELIZABLE FOLIATIONS OF CODIMENSION TWO
}

\author{
BY \\ LAWRENCE CONLON(1)
}

\begin{abstract}
We study framed foliations such that the framing of the normal bundle can be chosen to be invariant under the linear holonomy of each leaf. In codimension one there is a strong structure theory for such foliations due, e.g., to Novikov, Sacksteder, Rosenberg, Moussu. An analogous theory is developed here for the case of codimension two.
\end{abstract}

Introduction. Let $M$ be a smooth (i.e., $C^{\infty}$ ) connected manifold, $\mathscr{F}$ a smooth foliation of $M$ of codimension $q, Q$ the normal bundle of $\mathscr{F}$, and $F(Q)$ the normal frame bundle. If $H \subset G l_{q}$ is a Lie subgroup, a transverse $H$-structure for $\mathscr{F}$ will be a smooth reduction of $F(Q)$ to an $H$-bundle which is invariant under the natural parallelism along the leaves of $\mathscr{F}$. Such an $H$-reduction will be said to be "compatible" with $\mathscr{F}$ (more precise definitions appear in $\$ 1$ ). A foliation together with a choice of transverse $H$-structure will be called an $H$-foliation. In this way, for instance, the foliated manifolds with bundle-like metric of Reinhart [14] are interpreted simply as $O_{q}$-foliations and the Riemannian foliations of Pasternack [13] as foliations admitting a transverse $O_{q}$-structure, while transversally orientable foliations are those with a transverse $G l_{q}^{+}$-structure.

It seems reasonable to investigate the topological and geometric consequences of the existence of transverse $H$-structures for the various Lie groups $H$. In this paper we carry out such investigations for the extreme cases in which $H$ is discrete (transversally almost parallelizable foliations) and those in which $H=e$ is trivial (transversally parallelizable or $e$-foliations). As examples, the standard foliations of the torus $T^{n}$ induced by parallel $(n-q)$-planes in $\mathbf{R}^{n}$ are transversally parallelizable, while the foliation of an open Möbius strip by the curves parallel to the center circle is transversally almost parallelizable. We have a number of general results for such foliations valid in arbitrary codimension (cf. $\S \S 3$ and 4), but our strongest theorems are for codimension two and require $M$ to be compact.

In codimension one, $e$-foliations of compact manifolds are rather well understood. In this case, our condition is equivalent to the absence of limit cycles, a situation which has been studied by a variety of authors (e.g., [9], [11], [12]). In particular, a structure theorem due to Novikov [12] asserts that the universal cover $\hat{M}$ of $M$ will have the form $\hat{A} \times \mathbf{R}$ where $\hat{A}$ is the universal cover of the

Received by the editors May 3, 1973.

AMS (MOS) subject classifications (1970). Primary 57D30.

(1) Research partially supported by NSF Grant GP-20842.

Copyright $\odot$ 1974, American Mathematical Society 
typical leaf $A$ of $\mathscr{F}$. Furthermore, the inclusion map induces a monomorphism $\pi_{1}(A) \rightarrow \pi_{1}(M)$ and the quotient $\pi_{1}(M, A)=\pi_{1}(M) / \pi_{1}(A)$ is a free abelian group of finite rank $\geq 1$. If $\pi_{1}(M, A)=\mathrm{Z}$, then $M$ is fibered over $S^{1}$ with the leaves of $\mathscr{F}$ as fibers. If $\operatorname{rank}\left(\pi_{1}(M, A)\right) \geq 2$, then each leaf of $\mathscr{F}$ is everywhere dense in $M$. Much of this theorem remains true without requiring compactness and this is important for our purposes; hence a detailed treatment will be given in $\S 5$.

In higher codimension the absence of limit cycles does not imply any such result. For example, the Hopf fibration of $S^{3}$ is a codimension two foliation without limit cycles for which no analogue of the above result holds. The stronger assumption of transversal parallelizability, however, yields for codimension two a theorem quite similar to Novikov's. We state the theorem here and prove it in $\S \S 6$ and 7.

Main Theorem. Let $M$ be a compact connected $n$-manifold, $\mathscr{F}$ an e-foliation of $M$ of codimension two. Then the leaves of $\mathscr{F}$ are mutually diffeomorphic and the universal cover $\hat{M}$ of $M$ has the form $\hat{A} \times \mathbf{R}^{2}$ where $\hat{A}$ is the universal cover of the typical leaf

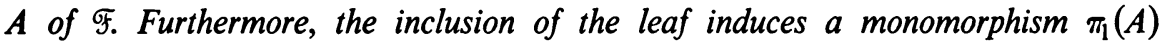
$\rightarrow \pi_{1}(M)$ onto a normal subgroup. The group $\pi_{1}(M, A)=\pi_{1}(M) / \pi_{1}(A)$ is nontrivial, contains no elements of finite order, and is not cyclic. In particular, if it is abelian, $\pi_{1}(M, A) \cong \mathbf{Z}^{r}, r \geq 2$. In this latter case, $r=2$ if and only if $\mathscr{F}$ is the foliation of $M$ by fibers of a smooth bundle $M \rightarrow T^{2}$, while $r>2$ implies either that each leaf $A$ of $\mathscr{F}$ is everywhere dense in $M$ or that the closures $\bar{A}$ are topological $(n-1)-$ manifolds which are the fibers of a topological bundle $M \rightarrow S^{1}$.

We remark that this theorem has no close analogue in codimension $\geq 3$. For instance $S^{3}$ foliated by points is transversally parallelizable since $S^{3}$ is parallelizable. A condition sufficient to guarantee the desired sort of result in higher codimension would be the existence of a Haefliger cocycle $[5]\left\{f_{\alpha}, g_{\alpha \beta}^{x}\right\}$ for $\mathscr{F}$ such that $d g_{\alpha \beta}^{x}$ is the identity of $G l_{q}$ for all $\alpha, \beta, x$. A $C^{0}$ version of this condition is satisfied by $e$-foliations of codimension two provided that $\pi_{1}(M, A)$ is abelian (cf. (10.7)).

The theorem is a bit more surprising than might appear at first sight. For instance, every nonsingular flow on $S^{3}$ gives a codimension two foliation with trivial normal bundle, but, by the first assertion in the theorem, there is no trivialization of that bundle which is compatible with the foliation. We list other easy but pleasant corollaries which will be proven in $\$ 9$.

Corollary A. If $M$ is a compact connected manifold with $\pi_{1}(M)$ finite, then $M$ does not admit a transversally almost parallelizable foliation of codimension $\leq 2$.

For this corollary one merely observes (\$3) that a transversally almost parallelizable foliation lifts to a transversally parallelizable one on the universal cover.

In the remaining corollaries we fix the hypothesis that $M$ is a compact connected $n$-manifold with an $e$-foliation $\mathcal{F}$ of codimension two. 
Corollary B. If $n \geq 5$ and $\pi_{k}(M)=0,2 \leq k \leq n-3$, then either $\hat{M}$ is contractible or the foliation is a fiber bundle $M \rightarrow T^{2}$, the fiber having universal cover a homotopy $(n-2)$-sphere.

The next two corollaries are variations on the same theme as Corollary B, merely incorporating special properties of dimensions 4 and 3.

Corollary C. If $n=4$, then either $\hat{M} \cong \mathbf{R}^{4}$ or the foliation is a fiber bundle over $T^{2}$ with fiber $S^{2}$ or the projective plane $P^{2}$.

Corollary D. If $n=3$, then $\hat{M} \cong \mathbf{R}^{3}$.

When $\pi_{1}(M)$ is abelian we have $\pi_{1}(M, A)$ free abelian and there result some fairly strong structure theorems as exemplified by our last two corollaries (also cf. (10.7)).

Corollary E. If $\pi_{1}(M)$ is abelian there is an integer $k \geq 2$ and, up to homotopy, a fibration $A \rightarrow M \rightarrow T^{k}$ where $A \rightarrow M$ is the one-one immersion of a leaf of $\mathscr{F}$.

Corollary F. If $\pi_{1}(M)$ is abelian and the leaves of $\mathscr{F}$ are of the form $T^{r} \times \mathbf{R}^{n-r-2}$, $0 \leq r \leq n-2$, then $M$ has the homotopy type of $T^{n}$.

Notations and conventions. As is customary, $\mathbf{R}$ will denote the real number system, $\mathbf{Z}$ the system of integers, and $\mathbf{Z}_{p}$ the integers $\bmod p$.

If $E$ is a smooth vector bundle, $\Gamma(E)$ will denote the vector space of smooth cross sections of $E$.

All manifolds are Hausdorff. Each component of a manifold will be assumed second countable, hence paracompact. If $M$ is a smooth manifold the tangent bundle of $M$ will be denoted by $T(M) . T_{x}(M)$ will denote the fiber of $T(M)$ at $x \in M$ and $\chi(M)$ will denote $\Gamma(T(M))$.

We remark that the smoothness assumptions governing all constructions in this paper can be relaxed to a class $C^{k}, k \geq 2$.

1. Transverse $H$-structures. Given a smooth codimension $q$ foliation $\mathscr{F}$ of a smooth connected manifold $M$, let $E$ be the subbundle of $T(M)$ consisting of the vectors tangent to the leaves of $\mathscr{F} . E$ is called the tangent bundle to $\mathscr{F}$ and $Q=T(M) / E$ is called the normal bundle to $\mathscr{F}$.

The foliation $\mathscr{F}$ gives rise to a collection $\left\{U_{\alpha}, f_{\alpha}\right\}_{\alpha \in A}$ where $\left\{U_{\alpha}\right\}_{\alpha \in A}$ is an open cover of $M$ and $f_{\alpha}: U_{\alpha} \rightarrow \mathbf{R}^{q}$ is a submersion constant along the connected components of the intersection of $U_{\alpha}$ with any leaf of $\mathscr{F}[4]$. Clearly

$$
f_{\alpha^{*}}: Q \mid U_{\alpha} \rightarrow T\left(\mathbf{R}^{q}\right)
$$

is a well-defined bundle map which is an isomorphism on fibers.

If $L$ is a leaf of $\mathscr{F}$ and $s:[a, b] \rightarrow L$ a path on $L$, then there is a natural parallel translation of vectors $v \in Q_{s(a)}$ along $s$. Indeed, find a subdivision $a=t_{0}<t_{1}$ $<\cdots<t_{r}=b$ and a set $\alpha_{0}, \alpha_{1}, \ldots, \alpha_{r-1} \in A$ such that $s_{i}=s \mid\left[t_{i}, t_{i+1}\right]$ lies on 
a connected component of $L \cap U_{\alpha_{i}}$. Then, if $v\left(t_{i}\right)$ has been defined $\left(v(a)=v\left(t_{0}\right)\right.$ $=v$ ), we choose for $t_{i} \leq t \leq t_{i+1}$ the unique vector $v(t) \in Q_{s(t)}$ such that

$$
f_{\alpha_{i}^{*}}(v(t))=f_{\alpha_{i}^{*}}\left(v\left(t_{i}\right)\right)
$$

as elements of $T_{f_{a}(s(t))}\left(\mathbf{R}^{q}\right)$. It is elementary that $v(b)$ obtained by this process depends only on $s$ and $v$, and not on the above choices of $t_{i}$ and $\alpha_{i}$. Indeed, it is evident that this parallelism is locally absolute; hence $v(b)$ depends only on the homotopy class of $s$ as a path on $L$ from $s(a)$ to $s(b)$. Clearly $v \mapsto v(b)$ defines a vector space isomorphism $\tau_{s}: Q_{s(a)} \rightarrow Q_{s(b)}$.

The above parallel translation of normal vectors to $\mathscr{F}$ along paths lying on a leaf of $\mathscr{F}$ will be referred to as the "natural parallelism along the leaves". If $F(Q)$ is the frame bundle of $Q$, then this natural parallelism is also defined for frames $\left(v_{1}, \ldots, v_{q}\right) \in F(Q)$.

(1.1) Definition. Let $H \subset G l_{q}$ be a Lie subgroup. A $\left(C^{k}\right)$ transverse $H$-structure for $\mathscr{F}$ is a $\left(C^{k}\right)$ reduction $P \subset F(Q)$ of $F(Q)$ to a principal $H$-bundle $P$ such that the natural parallelism along leaves always carries elements of $P$ to elements of $P$. Such an $H$-reduction is also said to be compatible with $\mathscr{F}$.

In this definition we may take $0 \leq k \leq \infty$, but the in the present paper we are assuming $k=\infty$.

Examples of such transverse $H$-structures have already been mentioned in the introduction. In the example of the open Möbius strip foliated by curves parallel to the center circle we have $H=\mathrm{Z}_{2}$. If $H$ is the trivial group $e$ (the case in which we say that the foliation is transversally parallelizable), we can find global sections $Z_{1}, \ldots, Z_{q}$ of $Q$ which are everywhere parallel along leaves (i.e. independently of choices of paths) and which give a basis of $Q_{x}, \forall x \in M$. If we only ask that $Q$ admit global sections $Z_{1}, \ldots, Z_{k}$ everywhere parallel along leaves and everywhere linearly independent, then $\mathscr{F}$ admits a transversal $G_{q-k^{-}}$ structure, where $G_{q-k}$ is the subgroup of $G l_{q}$ consisting of all matrices of the form

$$
\left[\begin{array}{ll}
I_{k} & 0 \\
B & A
\end{array}\right]
$$

where $A \in G l_{q-k}$.

Closely tied in with these notions is the concept of a basic connection on $Q$ as formulated by Bott [2, pp. 32-33]. Presuming familiarity with the standard definition of a connection on a smooth vector bundle (in terms of a Koszul operator $\nabla$ ), we can reformulate Bott's definition as follows.

(1.2) Definition. A connection $\nabla$ on $Q$ is a basic connection if, whenever $U \subset M$ is open, $X \in \Gamma(E \mid U)$, and $Y \in \Gamma(Q \mid U)$ is parallel along the leaves of $\mathscr{F} \mid U$, then $\nabla_{X} Y \equiv 0$.

(1.3) Lemma. If $\nabla$ and $\tilde{\nabla}$ are basic connections on $Q$, if $X \in \Gamma(E)$ and $Y \in \Gamma(Q)$, then $\nabla_{X} Y \equiv \tilde{\nabla}_{X} Y$. 
Proof. It is enough to work locally. Thus we obtain a local basis of parallel sections $Y_{1}, \ldots, Y_{q} \in \Gamma(Q \mid U)$ and we write $Y=\sum_{i=1}^{q} f_{i} Y_{i}$ where $f_{i} \in C^{\infty}(U)$. Then

$$
\nabla_{X} Y=\sum f_{i} \nabla_{X} Y_{i}+\sum X\left(f_{i}\right) Y_{i}=\sum X\left(f_{i}\right) Y_{i}
$$

and, similarly,

$$
\tilde{\nabla}_{X} Y=\Sigma X\left(f_{i}\right) Y_{i}
$$

Q.E.D.

(1.4) Lemma. Basic connections exist.

Proof. Use a Riemannian metric to represent

$$
T(M)=E \oplus Q
$$

Then any $X \in \mathcal{X}(M)$ decomposes uniquely into $X_{E}+X_{Q}$, the $E$ and $Q$ components respectively.

It is well known that connections always exist on smooth paracompact vector bundles; hence choose a connection $\tilde{\nabla}$ on $Q$. Define $\nabla$ on $Q$ by

$$
\nabla_{X} Y=\tilde{\nabla}_{X_{Q}} Y+\left[X_{E}, Y\right]_{Q}
$$

R-bilinearity in $(X, Y)$ and the property

$$
\nabla_{X}(f Y)=X(f) Y+f \nabla_{X} Y
$$

are obvious. Furthermore,

$$
\begin{aligned}
\nabla_{f X} Y & =\tilde{\nabla}_{f X_{Q}} Y+\left[f X_{E}, Y\right]_{Q} \\
& =f \tilde{\nabla}_{X_{Q}} Y+\left(f\left[X_{E}, Y\right]-Y(f) X_{E}\right)_{Q} \\
& =f \tilde{\nabla}_{X_{Q}} Y+f\left[X_{E}, Y\right]_{Q}=f \nabla_{X} Y .
\end{aligned}
$$

Finally, if $U \subset M$ is open, we can assume that the foliation on $U$ is given by a submersion $f: U \rightarrow \mathbf{R}^{q}$. If $Y \in \Gamma(Q \mid U)$ is parallel along leaves, then $f_{*} Y$ is well defined as a vector field on an open subset of $\mathbf{R}^{q}$. If $X \in \Gamma(E \mid U)$, then $f_{*} X=f_{*} X_{E} \equiv 0$, so

$$
f_{*}\left[X_{E}, Y\right]=\left[0, f_{*} Y\right] \equiv 0
$$

and $\left[X_{E}, Y\right] \in \Gamma(E \mid U)$. That is,

$$
\nabla_{X} Y=\left[X_{E}, Y\right]_{Q} \equiv 0 .
$$

Q.E.D. 
From these lemmas it follows that a connection $\nabla$ on $Q$ is basic iff for any $X \in \Gamma(E)$ and $Y \in \Gamma(Q)$,

$$
\nabla_{X} Y=[X, Y]_{Q}
$$

This is Bott's definition.

(1.5) Corollary. For any open $U \subset M, Y \in \Gamma(Q \mid U)$ is parallel along the leaves iff $[X, Y] \in \Gamma(E \mid U), \forall X \in \Gamma(E \mid U)$.

Here, of course, we continue to choose an identification $T(M)=E \oplus Q$.

From standard connection theory and our basic definition one can prove the following.

(1.6) Proposition. $\mathcal{F}$ admits a transverse $H$-structure iff $Q$ admits a basic connection with holonomy group contained in $H$.

For some purposes it is useful to reformulate (1.1) in a somewhat less concise fashion. We require a set $\left\{U_{\alpha}, f_{\alpha}\right\}_{\alpha \in A}$ as in the second paragraph of this section and, for each $\alpha \in A$, an $H$-reduction $P_{\alpha}$ of the frame bundle $F\left(T\left(\mathbf{R}^{q}\right)\right)$, all such that each $f_{\alpha^{*}}: F(Q) \mid U_{\alpha} \rightarrow F\left(T\left(\mathbf{R}^{q}\right)\right)$ restricts to a bundle map

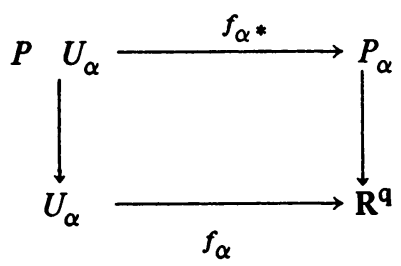

The equivalence of this with (1.1) is completely elementary. When we take $H=O_{q}$, this second formulation immediately gives Pasternack's definition of an $R$-foliation [13].

Using the second formulation one obtains, by analogy with Haefliger's $\Gamma_{q}$ structures [5], [6], [2], the notion of an $H \Gamma_{q}$-structure and constructs classifying spaces $B H \Gamma_{q}$.

2. Transverse $G_{q-1}$-structures. We recall from $\S 1$ that a transverse $G_{q-1^{-}}$ structure amounts to a nowhere zero $Y \in \Gamma(Q)$ which is everywhere parallel along leaves. Let $L_{Y} \subset Q$ be the line bundle generated by $Y$. As usual, we have selected a splitting $T(M)=E \oplus Q$, so $L_{Y}$ is a subbundle of $T(M)$.

(2.1) Proposition. $E \oplus L_{Y}$ is an integrable subbundle of $T(M)$.

Proof. If $X \in \Gamma(E)$, then $[X, Y] \in \Gamma(E)$ by (1.5). It follows that $\Gamma\left(E \oplus L_{Y}\right)$ is a Lie subalgebra of $\mathscr{X}(M)$. Q.E.D.

This proposition provides a foliation $\mathscr{F}_{Y}$ of codimension $q-1$, each leaf of which is foliated in codimension one by a collection of the leaves of $\mathscr{F}$. This is not an arbitrary codimension one foliation but is transversally parallelizable. 
Another useful and elementary consequence of $[\Gamma(E), Y] \subset \Gamma(E)$ is the following.

(2.2) Proposition. Let $Y$ as above be complete (e.g., this is automatic if $M$ is compact). Let $\varphi: \mathbf{R} \times M \rightarrow M$ be the flow generated by $Y$. Then the foliation $\mathcal{F}$ is invariant under $\varphi_{t}, \forall t \in \mathbf{R}$.

Indeed, for any $p \in M$ and $t \in \mathbf{R}$ we must have

$$
\varphi_{t^{*}}\left(E_{p}\right)=E_{\varphi_{1}(p)}
$$

and (2.2) follows directly from (2.3).

(2.4) Corollary. If $Y$ as above is complete and if $s_{1}, s_{2}$ are integral curves to $Y$ such that $s_{1}(0)$ and $s_{2}(0)$ lie on a common leaf $L_{0}$ of $\mathscr{F}$, then $s_{1}(t)$ and $s_{2}(t)$ lie on a common leaf $L_{t}$ of $\mathscr{F}, \forall t \in \mathbf{R}$.

Thus we can "parallel translate" integral curves to $Y$ along a leaf, the curves continuing to intersect the same leaves at the same parameter values. Indeed, in this way we sweep out the leaves of $\mathscr{F}_{\boldsymbol{Y}}$.

3. Transversally almost parallelizable foliations. We suppose there is an $H$ reduction $P \subset F(Q)$ compatible with the foliation, where $H$ is a discrete subgroup of $G l_{q}$. The bundle projection $\pi: P \rightarrow M$ is then a covering space with $H$ as the group of deck transformations. Since $P$ is invariant under the natural parallelism along leaves, it is practically tautologous to observe that the pull-back foliation $\pi^{-1}(\mathscr{F})$ on $P$ is transversally parallelizable. Indeed, $\pi^{-1}(F(Q)) \subset P$ $\times F(Q)$ is the normal frame bundle of $\pi^{-1}(\mathscr{F})$ and the tautologous section

$$
s: P \rightarrow \pi^{-1}(F(Q)), \quad s(p)=(p, p),
$$

is parallel along all the leaves of $\pi^{-1}(\mathscr{F})$. For the following proposition it will be enough to take $\tilde{M}$ to be a connected component of $P$.

(3.1) Proposition. If $\mathscr{F}$ is transversally almost parallelizable, then there is a connected covering space $\pi: \tilde{M} \rightarrow M$ such that $\pi^{-1}(\mathscr{F})$ is transversally parallelizable.

In particular, if $\hat{\pi}: \hat{M} \rightarrow M$ is the universal covering, $\hat{\pi}$ factors into

$$
\hat{M} \stackrel{\pi}{\rightarrow} \tilde{M} \rightarrow M
$$

and so $\hat{\pi}^{-1}(\mathscr{F})$ is transversally parallelizable.

One of the aims of this paper is to show that a compact manifold with finite fundamental group does not admit a codimension two transversally almost parallelizable foliation. By the above discussion it will be enough to show that a compact simply connected manifold does not admit a codimension two transversally parallelizable foliation. This, of course, is an immediate corollary of the Main Theorem as stated in the introduction. 
4. Transversally parallelizable foliations of arbitrary codimension. In the case in which $\mathscr{F}$ is an $e$-foliation the phenomena of $\$ 2$ happen in "all directions". More precisely, let $\left(Y_{1}, \ldots, Y_{q}\right)$ be a smooth cross section of $F(Q)$ which is everywhere parallel along the leaves. This section, which is simply a transverse $e$-structure for F, spans a $q$-dimensional vector subspace $V \subset \Gamma(Q)$ consisting entirely of fields everywhere parallel along the leaves. Each $Z \in V$ will be said to belong to the given transverse $e$-structure. Clearly $Z \in V$ and $Z \neq 0$ implies $Z_{p} \neq 0, \forall p$ $\in M$, hence the theory of $\$ 2$ gives rise to a foliation $\mathscr{F}_{Z}$ of codimension $q-1$, each leaf of which is $e$-foliated by some of the leaves of $\mathscr{F}$. If $Z$, identified as a field on $M$ (via a Riemannian metric), is complete, the foliation $\mathscr{F}$ is invariant under the flow generated by $Z$ and the integral curves to $Z$ parallel translate "nicely" along leaves of $\mathscr{F}$.

We choose once and for all a Riemannian metric on $M$ relative to which $Q$ is realized as the orthogonal complement of $E$ in $T(M)$ and such that $\left\langle Y_{i}, Y_{j}\right\rangle \equiv \delta_{i j}$, $1 \leq i, j \leq q$.

(4.1) Definition. An $e$-foliation as above is transversally complete if every $Z \in V$ is complete as a vector field on $M$.

Recall also the following standard terminology for any foliation $\mathscr{F}$ of $M$. If $p \in M$, a coordinate neighborhood $U$ of $p$ is called a product neighborhood if for some $a>0$ the coordinates $x_{i}$ on $U$ all range over $(-a, a)$ and if the foliation $\mathscr{F} \mid U$ naturally induced by $\mathscr{F}$ has as leaves exactly the level surfaces $x_{i}=$ constant, $1 \leq i \leq q$. Each leaf of $\mathscr{F} \mid U$ is, of course, a connected component of $L \cap U, L$ some leaf of $\mathscr{F}$, and is called a local leaf of $\mathscr{F}$. A leaf $L$ of $\mathscr{F}$ is called regular if, for each $p \in L, \exists$ a product neighborhood $U$ of $p$ such that $U \cap L^{\prime}$ is at most one local leaf, $\forall$ leaf $L^{\prime}$ of $\mathscr{F} . L$ is called proper if, for each $p \in L, \exists$ a product neighborhood $U$ of $p$ such that $U \cap L$ is exactly one local leaf. We remark that regular implies proper, but not conversely. Indeed, the foliation of an open Möbius strip by curves parallel to the center circle has the center circle as a leaf which is proper but not regular.

Returning to the assumption that $\mathscr{F}$ has a transverse $e$-structure $\left(Y_{1}, \ldots, Y_{q}\right)$, we remark on a simple but useful construction. Let $x \in M, a=\left(a_{1}, \ldots, a_{q}\right) \in \mathbf{R}^{q}$, and let $s_{a}$ be the integral curve to $Z=\sum a_{i} Y_{i}$ such that $s_{a}(0)=x$. Then $\exp _{x}(a)=s_{a}(1)$ defines a map

$$
\exp _{x}: \mathbf{R}^{q} \rightarrow M .
$$

By standard arguments from the theory of ordinary differential equations, $\exp _{x}$ is smooth and is regular at $0 \in \mathbf{R}^{q}$, hence defines a small transverse open $q$-disk $D_{x}$ centered at $x$. In particular, all leaves passing suitably near $x$ can be reached by an integral curve to some $Z \in V$ starting at $x$.

With these preliminary remarks out of the way, we are prepared to prove some propositions of fundamental importance. 
(4.2) Proposition. For any leaf $L$ of a transversally complete e-foliation of $M$, the following are equivalent:

(1) $L$ is closed in $M$;

(2) $L$ is proper;

(3) $L$ is regular.

Proof. (1) $\Rightarrow(2)$ holds for any foliation (cf. [3, pp. 109-110]).

In order to show (2) $\Rightarrow(3)$, suppose $L$ not regular and choose $p \in L$. Let $U$ be any product neighborhood of $p$ and let $L_{0}$ be the component of $p$ in $L \cap U$. By assumption, for any $\varepsilon>0$ there is a leaf $L^{\prime}$ such that $L^{\prime} \cap U$ has at least two components both within $\varepsilon$ of $L_{0}$. Call these components $L_{1}^{\prime}$ and $L_{2}^{\prime}$ respectively. Let $s:[0,1] \rightarrow U$ be a segment of integral curve to suitable $Z \in V$ such that $s(1)=p, s(0) \in L_{1}^{\prime}$ (such $s$ exists if $\varepsilon$ is chosen sufficiently small). Parallel translate $s$ along $L^{\prime}$ so as to obtain $\tilde{s}$, also integral to $Z$ with $\tilde{s}(0) \in L_{2}^{\prime}$. If $\varepsilon$ is sufficiently small we may assume $\tilde{s}[0,1] \subset U$. Then if $\tilde{s}(1) \in L_{0}$ we may assume $\tilde{s}(1)=p$; hence $\tilde{s}=s$ contradicting $L_{1}^{\prime} \neq L_{2}^{\prime}$. Thus $\tilde{s}(1)$ lies on a component of $L \cap U$ other than $L_{0}$. $U$ being arbitrary, $L$ cannot be proper.

In order to prove (3) $\Rightarrow(1)$, suppose $L$ not closed in $M$. Let $x \in \bar{L} \backslash L$ and let $\left\{x_{n}\right\}$ be a sequence in $L$ with $x_{n} \rightarrow x$ in $M$. Choose a small product neighborhood $U$ of $x$ and assume $\left\{x_{n}\right\} \subset U$. Since $x \notin L$, no generality is lost in assuming a sequence of distinct components $L_{n}$ of $L \cap U$ with $x_{n} \in L_{n}, n=1,2, \ldots$. Let $L^{\prime}$ be the leaf through $x$ and $L_{0}^{\prime}$ the component of $x$ in $L^{\prime} \cap U$. Then $L_{n} \rightarrow L_{0}^{\prime}$ uniformly. For any sufficiently small $\varepsilon>0$ find $n$ and an integral curve $s:[0,1] \rightarrow U$ to suitable $Z \in V$ such that $s$ has length $<\varepsilon$ and $s(0) \in L_{n}$, $s(1) \in L_{0}^{\prime}$. By having chosen $\varepsilon$ small enough, we guarantee that $s$ parallel translates along $L$ to an integral curve $\tilde{s}$ to $Z$ with $\tilde{s}[0,1] \subset U, \tilde{s}(0) \in L_{1}$.

Since $Z=\sum a_{i} Y_{i}$ has constant norm $\|Z\|=\sqrt{a_{1}^{2}+\cdots+a_{q}^{2}}$, this is the length of both $s$ and $\tilde{s}$. Since $\tilde{s}(1) \in L^{\prime}$, it follows that there is a component of $L^{\prime} \cap U$ of distance less than $\varepsilon$ from $L_{1} . \varepsilon>0$ was arbitrarily small, so $L$ cannot be regular. Q.E.D.

(4.3) Proposition. Let $\mathcal{F}$ be a transversally complete e-foliation of the connected manifold $M$. Then any two leaves of $\mathscr{F}$ are diffeomorphic and if some leaf is closed in $M$ they all are.

Proof. The flows generated by elements of $V$ provide enough diffeomorphisms of $M$, all leaving $\mathscr{F}$ invariant, to carry any leaf to any other. The desired consequences are immediate. Q.E.D.

These results enable us to prove the following proposition which will be of fundamental importance.

(4.4) Proposition. Let $\mathscr{F}$ be a transversally complete e-foliation of a connected manifold $M$. If $\mathcal{F}$ admits a closed leaf then there is a fiber bundle $p: M \rightarrow N$ where $N$ is parallelizable and $\mathcal{F}$ is the foliation of $M$ by the fibers of $p$. 
(It was pointed out by the referee that this result is closely related to the theorem of Ehresmann that a submersion of a compact manifold is a fiber bundle; cf. Rend. Mat. 10 (1951), p. 68.)

Proof. By (4.3), all leaves are closed; hence by (4.2) all leaves are regular. Let $L$ be a leaf, $x \in L$, and let $D_{x}$ be the small transverse open $q$-disk centered at $x$ which is defined by the map $\exp _{x}: \mathbf{R}^{q} \rightarrow M$. By the regularity of $L, D_{x}$ can be assumed to intersect any leaf at most once. Since $D_{x}$ is the union of radial curves integral to fields in $V, D_{x}$ parallel translates along $L$. If $D_{y}$ is a translate of $D_{x}$, some $y \in L$, then $D_{y}$ meets the same leaves as $D_{x}$ at the same parameter values $\left(a_{1}, \ldots, a_{q}\right) \in \mathbf{R}^{q}$. Also, if $z \in D_{x} \cap D_{y}$, there is $\left(a_{1}, \ldots, a_{q}\right)=a$ with $z$ $=\exp _{x}(a)=\exp _{y}(a)$; hence the curves $\exp _{x}(t a)$ and $\exp _{y}(t a)$ are integral to the same $Z=\sum a_{i} Y_{i}$ and both pass through $z$ at $t=1$; hence the curves are the same and $x=y$. It follows that there is a neighborhood $U$ of $L$ in which the foliation is diffeomorphic to the foliation of $D_{x} \times L$ by leaves of the form pt . $\times L$. Let $L$ and $L^{\prime}$ be distinct leaves, $x \in L$. Clearly $D_{x}$ can so be chosen that $\bar{D}_{x} \cap L^{\prime}=\varnothing$ and $\bar{D}_{x}$ is compact. Since $L^{\prime}$ is closed it lies at a positive distance $\eta$ from $\bar{D}_{x}$. For $y \in L^{\prime}$, choose $D_{y}$ so that the radial curves $\exp _{y}(t a)$ spanning $D_{y}$ have lengths $\sqrt{a_{1}^{2}+\cdots+a_{q}^{2}}<\eta$. Thus we obtain neighborhoods of $U$ of $L$ and $U^{\prime}$ of $L^{\prime}$ as above such that $U \cap U^{\prime}=\varnothing$. It follows that the leaf space $M / \mathscr{F}$ is a smooth Hausdorff $q$-manifold $N$ and the natural projection $p: M \rightarrow N$ a smooth fiber bundle with fibers the leaves of $\mathscr{F}_{\text {. The frame }}\left(Y_{1}, \ldots, Y_{q}\right)$ consists of normal fields everywhere parallel along the leaves; hence each $p_{*}\left(Y_{i}\right)$ is a well-defined field on $N$ and $\left(p_{*}\left(Y_{1}\right), \ldots, p_{*}\left(Y_{q}\right)\right)$ is a global frame field on $N$. That is, $N$ is parallelizable. Q.E.D.

(4.5) Corollary. Let $M$ be a compact connected 3-manifold, $\pi_{1}(M)$ finite. If $M$ admits an e-foliation by curves, then no leaf is a circle.

Proof. By compactness the $e$-foliation is automatically transversally complete. By (4.4), if some leaf is a circle then all are circles and we have a fibration $S^{1} \rightarrow M \rightarrow T^{2}$ (since $T^{2}$ is the only compact connected paralellizable 2-manifold). By the exact homotopy sequence we contradict $\pi_{1}(M)$ finite. Q.E.D.

Remark. By (4.5) any $e$-foliation of $S^{3}$ by curves would provide a $C^{\infty}$ counterexample to the Seifert conjecture (for a $C^{1}$ counterexample, cf. [17]). We will see, however, that no such $e$-foliation exists.

5. $e$-foliations of codimension one. Although our principal interest in this paper is the codimension two case, much of what is already known about $e$-foliations of codimension one can be obtained quite easily using our present methods. Since these results have important applications to the codimension two case, it seems worthwhile to develop them carefully here.

(5.1) Proposition. Let $M$ be connected, $\mathscr{F}$ a transversally complete e-foliation of codimension one. If $\mathcal{F}$ does not have a closed leaf, then each leaf is everywhere dense in $M$. 
Proof. Suppose the leaves are not closed and that $L$ is a leaf which is not everywhere dense. Then $W=M \backslash \bar{L}$ is a nonempty open set. $W$ cannot also be closed since $M$ is connected. Let $x \in \bar{W} \backslash W$ and let $U$ be a product neighborhood of $x$. $U$ meets both $W$ and $\bar{L}$. Let $y \in W \cap U$ and let $s$ be a transversal in $U$ with $s(0)=y \cdot \bar{L}$ is a union of leaves, hence so is $W$, and the codimension one hypothesis allows us to assume that $s$ meets both $W$ and $\bar{L}$. Let $t_{0}>0$ be the smallest parameter value such that $s\left(t_{0}\right) \in \bar{L}$. Let $L^{\prime}$ be the leaf through $s\left(t_{0}\right)$. $L^{\prime}$ is not closed; hence by (4.2) it is not proper, so $L^{\prime} \cap U$ has a countable infinity of components. Let $L_{0}^{\prime}$ be the component of $s\left(t_{0}\right)$ in $L^{\prime} \cap U$. We claim that the other components must lie on both sides of $L_{0}^{\prime}$. Indeed, if $\left\{L_{n}^{\prime}\right\}$ is a sequence of components approaching $L_{0}^{\prime}$ from one side, consider an integral curve to $Z \in V$ from some $L_{n}^{\prime}$ to $L_{0}^{\prime}$ lying in $U$. It must properly cross some $L_{m}^{\prime}$; hence parallel translation along $L^{\prime}$ will move it to an integral curve of $Z$ properly crossing $L_{0}^{\prime}$; hence its two ends lie on opposite sides of $L_{0}^{\prime}$ and both lie on $L^{\prime} \cap U$. Thus we find $t_{1} \in\left(0, t_{0}\right)$ such that $s\left(t_{1}\right) \in L^{\prime} \subset \bar{L}$, contradicting the minimality of $t_{0}$. Q.ED.

The following well-known consequence is usually proven using number theory.

(5.2) Corollary. Foliate $T^{n}$ by foliating $\mathbf{R}^{n}$ with parallel $(n-1)$-planes. Suppose that the plane through 0 does not meet the integer lattice of $\mathbf{R}^{n}$ in any other point. Then each leaf is everywhere dense in $T^{n}$.

Proof. The foliation is clearly an $e$-foliation of $T^{n}$ of codimension one and is transversally complete. By the hypothesis, at least one leaf is not compact, hence is not closed. The conclusion follows by (4.3) and (5.1). Q.E.D.

(5.3) Proposition. If $\mathscr{F}$ is a codimension one transversally complete e-foliation of a connected and simply connected manifold $M$, then $M \cong A \times \mathbf{R}$ where the leaves of $\mathscr{F}$ become identified with the manifolds $A \times \mathrm{pt}$.

Proof. Let $L$ be a leaf of $\mathscr{F}$. If $L$ is not closed, then by (4.2) it is not proper. Thus there is a product neighborhood $U$ such that $L \cap U$ has more than one component. Construct a closed piecewise $C^{\infty}$ curve on $M$ consisting of a transverse segment in $U$ joining two components of $L \cap U$ and a segment lying on $L$. By a standard technique (cf. [12, p. 269]) modify this closed curve to obtain a closed $C^{\infty}$ transversal $\sigma$ to $\mathscr{F}$. Since $M$ is simply connected, $\sigma$ is homotopic to a constant; hence Haefliger's lemma [4, Proposition 4.2] shows that some leaf of $\mathscr{F}$ has nontrivial holonomy $[4, \S 2]$. This contradicts the assumption that $\mathscr{F}$ is an $e$ foliation (cf. (2.4)), so it follows that every leaf of $\mathscr{F}$ is closed in $M$. By (4.4) it follows that the foliation is a fiber bundle over $S^{1}$ or $\mathbf{R}$. Since $\pi_{1}(M)=0$, it must be a bundle over $\mathbf{R}$. Since $\mathbf{R}$ is contractible, the fibration is a product. Q.E.D.

Before coming to the main theorem of this section, we cite a deep result of Sacksteder [16, Theorem 6]. He shows that a codimension one foliation of compact $M$ such that each leaf has finite holonomy admits a transverse $O(1)$ - 
structure, at least after the introduction of a possibly new smooth structure on $\boldsymbol{M}$ (relative to which the foliation remains smooth). If the foliation is transversally orientable it is clear that one obtains a transverse $S O(1)$-structure.

(5.4) Theorem (Sacksteder). Let $M$ be a compact manifold, $\mathscr{F}$ a codimension one foliation without limit cycles (i.e., such that each leaf has trivial holonomy). Then, in a possibly new differentiable structure for $M, \mathcal{F}$ is a transversally complete e-foliation.

By (5.4) the hypotheses of the following theorem are verified when $M$ is compact and $\mathscr{F}$ is a codimension one foliation without limit cycles. These are the hypotheses in [12, Theorem 5.1], so the following is a mild generalization of that theorem. We will need the more general result for the proof of our Main Theorem. (It should be remarked that the essentials of a very elegant proof of (5.5) using differential forms will be found in [15, pp. 171-172] and in [9]. The point of view taken in our proof, however, continues to be fruitful in codimension two, as does not seem true of the differential forms approach.)

(5.5) Theorem. Let $\mathcal{F}$ be a codimension one transversally complete e-foliation of a connected manifold $M$. Then the universal cover of $M$ has the form $\hat{M} \cong \hat{A} \times \mathbf{R}$ where $\hat{A}$ is the universal cover of the typical leaf $A$ of $F$. The inclusion of a leaf induces a monomorphism $\pi_{1}(A) \rightarrow \pi_{1}(M)$ onto a normal subgroup and, if $M$ is compact, $\pi_{1}(M, A)=\pi_{1}(M) / \pi_{1}(A)=\mathbf{Z}^{r}, r \geq 1$. Furthermore, $r=1$ implies that the foliation is a smooth bundle over $S^{1}$, while $r>1$ implies that each leaf of $\mathscr{F}$ is everywhere dense in $M$.

Proof. Clearly the lifted foliation $\hat{\mathscr{F}}$ of $\hat{M}$ is also a transversally complete $e$ foliation, so (5.3) shows that $\hat{M} \cong \hat{A} \times \mathbf{R}$ where the leaves of $\hat{\mathscr{F}}$ are of the form $\hat{A} \times t$. Since $\hat{A}$ must be simply connected it is the universal cover of the typical leaf $A$ of $\mathscr{F}$. It follows immediately that $\pi_{1}(A) \rightarrow \pi_{1}(M)$ is one-one.

Realize $\pi_{1}(M)$ as the group of covering transformations on $\hat{M}$. The $e$-structure of $\mathscr{F}$ defines a transverse flow $\varphi$ and the lifted flow $\hat{\varphi}$ leaves $\hat{\mathscr{F}}$ invariant. Evidently $\hat{\varphi}$ commutes with the elements of $\pi_{1}(M)$ and can be used to reparametrize $\hat{M}=\hat{A} \times \mathbf{R}$ so that $\hat{\varphi}_{t}(\hat{A} \times 0)=\hat{A} \times t, \forall t \in \mathbf{R}$. Thus we obtain a representation $p: \pi_{1}(M) \rightarrow \operatorname{Diff}^{+}(\mathbf{R})$, the image of which is a group of translations. For $\alpha \in \pi_{1}(M), p(\alpha)$ has a fixed point if and only if $p(\alpha)$ is the identity; hence the image of $\pi_{1}(A)$ in $\pi_{1}(M)$ is the normal subgroup $\operatorname{Ker}(p)$. If $M$ is compact, $\pi_{1}(M)$ is finitely generated as is $\pi_{1}(M, A)=\pi_{1}(M) / \pi_{1}(A)$, so this latter group is $\mathbf{Z}^{r}, r \geq 0$. But $r=0$ would imply $M \cong A \times \mathbf{R}$ contradicting compactness. Evidently the leaves of $\mathscr{F}$ are closed in $M$ if and only if $r=1$, so the final assertions of the theorem follow from (4.4) and (5.1). Q.E.D.

6. The universal cover of codimension two $e$-foliations. Throughout this section and the next we assume $M$ compact and connected and we let $\mathscr{F}$ be a codimension two $e$-foliation of $M$ (necessarily transversally complete by the compactness of $M)$ with $\left(Y_{1}, Y_{2}\right)$ the transverse $e$-structure. As usual, $V$ denotes 
$\operatorname{span}_{R}\left\{Y_{1}, Y_{2}\right\}$ and, for each nonzero $Z \in V, \mathscr{F}_{Z}$ denotes the codimension one foliation generated by $\mathscr{F}$ and $Z$.

We recall Novikov's concept of "cycles which are limitwise homotopic to zero on the right or left" [12, pp. 277-278]. This concept is defined for codimension one foliations and refers to a closed loop on a leaf, homotopically nontrivial on that leaf, which determines trivial holonomy on at least one side of the leaf ("right" or "left") and so can be displaced to all nearby leaves on that side, which displaced loops, finally, are all nullhomotopic on their leaves. Following a prevalent trend (cf. [10], [11]) we will call these "vanishing cycles".

(6.1) Proposition. Let $0 \neq Z \in V$. Then $\mathscr{F}_{Z}$ admits no vanishing cycles.

Proof. Let $L$ be a leaf of $\mathscr{F}_{Z}, x \in L, \sigma$ a closed loop on $L$ based at $x$, [ $\left.\sigma\right]$ the corresponding element of $\pi_{1}(L, x)$.

Suppose that $\sigma$ is smooth and transversal to the foliation $\mathscr{F} \mid L$ of $L$ by leaves of $\mathscr{F} . \mathscr{F} \mid L$ is an $e$-foliation of $L$, necessarily transversally complete since $\mathscr{F}$ is transversally complete. Thus by (5.5) the universal cover $\hat{L}$ of $L$ has the form $\hat{A} \times \mathbf{R}$ where $\hat{A}$ is the universal cover of the typical leaf $A$ of $\mathscr{F} \mid L . \sigma$ lifts to a curve $\hat{\sigma}$ transversal to the leaves $\hat{A} \times$ pt. It follows that $\hat{\sigma}(0)$ and $\hat{\sigma}(1)$ lie on different leaves so that $\hat{\sigma}(0) \neq \hat{\sigma}(1)$; hence $[\sigma] \neq 0$. A small displacement (if it exists) of such $\sigma$ to a nearby leaf $L^{\prime}$ will again be transversal to $\mathscr{F} \mid L^{\prime}$, hence will not be homotopically trivial on $L^{\prime}$.

Suppose, then, that $\sigma$ is not base point homotopic on $L$ to a transversal to $\mathscr{F} \mid L$. Then $\hat{\sigma}$ must begin and end on the same $\hat{A} \times \mathrm{pt}$, so $\hat{\sigma}$ is fixed endpoint homotopic to a path in $\hat{A} \times$ pt. Assuming, therefore, that $\sigma$ lies entirely on a leaf $A$ of $\mathscr{F} \mid L$, we see that a small displacement to a nearby leaf $L^{\prime}$ of $\mathscr{F}_{Z}$ can be produced by the flow associated to a suitable $X \in V$ and the displaced curve $\sigma^{\prime}$ lies on a leaf $A^{\prime}$ of $\mathscr{F} \mid L^{\prime}$. By $(5.5), \pi_{1}(A) \rightarrow \pi_{1}(L)$ is one-one as is $\pi_{1}\left(A^{\prime}\right) \rightarrow \pi_{1}\left(L^{\prime}\right)$, so, if $[\sigma]$ is

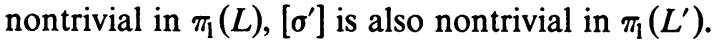

Thus we have shown in all cases that there is no vanishing cycle on any $L$. Q.E.D.

(6.2) Corollary. Let $Z \in V, L$ a leaf of $\mathscr{F}_{Z}, x \in L$. Then the inclusion of $L$ induces a monomorphism $\pi_{1}(L, x) \rightarrow \pi_{1}(M, x)$.

Proof. By [12, Theorem 6.1], the existence of a leaf $L$ such that $\pi_{1}(L) \rightarrow \pi_{1}(M)$ has nontrivial kernel would imply the existence of a vanishing cycle on some leaf of $\mathscr{F}_{Z}$. This contradicts the above proposition. Q.E.D.

(6.3) Corollary. If $A$ is a leaf of $\mathscr{F}, a \in A$, then the leaf inclusion induces $a$ monomorphism $\pi_{1}(A, a) \rightarrow \pi_{1}(M, a)$.

Proof. $A \subset L$ where $L$ is a leaf of $\mathscr{F}_{Z}, 0 \neq Z \in V$. By $(5.5), \pi_{1}(A, a) \rightarrow \pi_{1}(L, a)$ is one-one, and, by (6.2), $\pi_{1}(L, a) \rightarrow \pi_{1}(M, a)$ is one-one. The composition of these monomorphisms is $\pi_{1}(A, a) \rightarrow \pi_{1}(M, a)$. Q.E.D.

Let $\pi: \hat{M} \rightarrow M$ be the universal covering. $\hat{M}$ is $e$-foliated by $\hat{\mathscr{F}}$, the pull-back 
of $\mathscr{F}$ by $\pi$, and $\hat{\mathscr{F}}$ is transversally complete. By (6.3), each leaf $\hat{A}$ of $\hat{\mathscr{F}}$ is the universal cover of a leaf $A=\pi(\hat{A})$ of $\mathscr{F}_{\text {. Let }}\left(\hat{Y}_{1}, \hat{Y}_{2}\right)$ be the transverse $e$-structure for $\hat{F}, \hat{V}=\operatorname{span}_{\mathbf{R}}\left\{\hat{Y}_{1}, \hat{Y}_{2}\right\}$.

(6.4) Proposition. Each leaf of $\hat{\mathscr{F}}$ is closed in $\hat{M}$.

Proof. Let $\hat{A}$ be a leaf of $\hat{\mathscr{F}}$. If $\hat{A}$ is not closed in $\hat{M}$, it is not proper (by (4.2)). Thus there is $0 \neq \hat{X} \in \hat{V}$ and an integral curve $\sigma:[0,1] \rightarrow \hat{M}$ to $\hat{X}$ such that $\sigma(0)$ and $\sigma(1)$ both $\in \hat{A}$. If $X=\pi_{*}(\hat{X}) \in V$, consider $\mathscr{F}_{X}$. By the above remarks, there is a leaf $L$ of $\mathscr{F}_{X}$ and a covering leaf $\hat{L}$ of $\hat{\mathscr{F}}_{\mathcal{X}}$ such that $\hat{A} \subset \hat{L} \subset \hat{M}$ and $\operatorname{Im}(\sigma) \subset \hat{L}$. But $\hat{L} \cong \hat{A} \times \mathbf{R}$ by (5.5) and (6.2) and $\sigma$ is a transversal to $\hat{\mathscr{F}} \mid \hat{L}$, so it is impossible that $\sigma(0)$ and $\sigma(1)$ lie on the same leaf $\hat{A}$ of $\hat{\mathscr{F}}$. This contradiction shows that $\hat{A}$ is closed in $\hat{M}$. Q.E.D.

(6.5) Corollary. $\hat{M} \cong \hat{A} \times \mathbf{R}^{2}$ where $\hat{A}$ is the universal cover of the typical leaf of $\mathscr{F}$ and the leaves of $\hat{\mathscr{F}}$ become identified with the sets $\hat{A} \times \mathrm{pt}$.

Proof. By (4.4) and (6.4), there is a fibration $\hat{M} \rightarrow N$ with fibers the leaves of $\hat{\mathscr{F}}$ and $N$ a parallelizable 2 -manifold. Since $\hat{M}$ is simply connected, the exact homotopy sequence shows that $N$ is also simply connected. By the standard classification of compact surfaces, the triangulability of separable surfaces (open or closed), and the remark at the bottom of p. 104 of [1], the only simply connected 2-manifolds are $S^{2}$ and $\mathbf{R}^{2}$, of which only $\mathbf{R}^{2}$ is parallelizable. Since $\mathbf{R}^{2}$ is contractible, the fibration must be a product. Q.E.D.

7. The covering transformations of $\hat{M}$. As usual, let $\pi_{1}(M)$ denote the group of covering transformations of $\hat{M} \cong \hat{A} \times \mathbf{R}^{2} \cdot \pi_{1}(M)$ maps leaves of $\hat{\mathscr{F}}$ to leaves of $\hat{\mathscr{F}}$ and preserves the $e$-structure. In particular, this defines a representation.

$$
\rho: \pi_{1}(M) \rightarrow \operatorname{Diff}^{+}\left(\mathbf{R}^{2}\right) .
$$

(7.1) Proposition. Let $\alpha \in \pi_{1}(M)$. Then $\rho(\alpha)$ has a fixed point in $\mathbf{R}^{2}$ if and only if $\rho(\alpha)=$ identity.

Proof. Let $p \in \mathbf{R}^{2}$ with $\rho(\alpha)(p)=p$. Let $\hat{A}_{p}$ be the leaf of $\hat{\mathscr{F}}$ over $p$, let $0 \neq \hat{Z} \in \hat{V}$, and let $\hat{L}_{p}$ be the leaf of $\hat{\mathscr{F}}_{\boldsymbol{Z}}$ containing $\hat{A}_{p}$. $\alpha$ leaves $\hat{\mathscr{F}}_{\boldsymbol{Z}}$ invariant; hence $\alpha\left(\hat{L}_{p}\right)=\hat{L}_{p}$, so $\alpha$ restricts to a covering transformation for the universal cover $\hat{L}_{p} \rightarrow L_{p}=\pi\left(\hat{L}_{p}\right)$. As in the proof of (5.5), the fact that $\alpha\left(\hat{A}_{p}\right)=\hat{A}_{p}$ implies that $\alpha$ maps each leaf of $\hat{\mathscr{F}} \mid \hat{L}_{p}$ to itself. Since $0 \neq \hat{Z} \in \hat{V}$ was arbitrary, it follows that $\rho(\alpha)$ leaves a whole neighborhood of $p$ in $\mathbf{R}^{2}$ pointwise fixed, so the fixed point set of $\rho(\alpha)$ is open and nonempty. On the other hand, the fixed point set of $\rho(\alpha)$ must be closed, so this set is all of $\mathbf{R}^{2}$. Q.E.D.

Let $[\sigma] \in \pi_{1}(A, a) \subset \pi_{1}(M, a)$ for some leaf $A$ of $\mathscr{F}, a \in A$. Let $\hat{A}$ be a leaf of $\hat{\sigma}$ covering $A, \hat{a} \in \hat{A}$ such that $a=\pi(\hat{a})$. Let $\hat{\sigma}$ be the unique lift of $\sigma$ such that $\hat{\sigma}(0)=\hat{a}$. Then $\hat{\sigma}(1)=\alpha(\hat{a})$ for some covering transformation $\alpha$ of $\hat{M}$. Since $\alpha(A)=A$, we see that $\alpha \in \operatorname{Ker}(\rho)$ by (7.1). Conversely, if $\alpha \in \operatorname{Ker}(\rho)$, then $\alpha$ 
leaves $\hat{A}$ invariant and any curve on $\hat{A}$ from $\hat{a}$ to $\alpha(\hat{a})$ projects under $\pi$ to a representative of an element of $\pi_{1}(A, a) \subset \pi_{1}(M, a)$. We have proven the following fact.

(7.2) Proposition. Under the identification (via the choice of $\hat{a})$ of $\pi_{1}(M, a)$ with the group of covering transformations $\pi_{1}(M), \pi_{1}(A, a) \subset \pi_{1}(M, a)$ is exactly the subgroup identified with $\operatorname{Ker}(\rho)$.

Thus we will designate $\operatorname{Ker}(\rho)$ by $\pi_{1}(A)$ where $A$ denotes the generic leaf of $\mathscr{F}$. One sees, therefore, that there is little need to distinguish $\pi_{1}(M)$ from $\pi_{1}(M, a)$ and $\pi_{1}(A)$ from $\pi_{1}(A, a)$.

(7.3) Corollary. $\pi_{1}(A)$ is a normal subgroup of $\pi_{1}(M)$.

Our next object of study will be the group $\pi_{1}(M) / \pi_{1}(A)$. We remark that this group identifies naturally with the relative homotopy set $\pi_{1}(M, A)$ because of the exact sequence

$$
0 \rightarrow \pi_{1}(A, a) \rightarrow \pi_{1}(M, a) \rightarrow \pi_{1}(M, A, a) \rightarrow \pi_{0}(A, a)=0 .
$$

We also remark that there is an intermediate covering space $\tilde{M} \rightarrow M$ with $\pi_{1}(M, A)$ as group of covering transformations. $\tilde{M}$ is obtained from $\hat{M}$ by dividing out the action of $\pi_{1}(A)$; hence $\tilde{M} \cong A \times \mathbf{R}^{2}$.

(7.4) Lemma. $\pi_{1}(M, A) \neq 0$.

Proof. Otherwise $M=\tilde{M}=A \times \mathbf{R}^{2}$, contradicting the fact that $M$ is compact. Q.E.D.

(7.5) Lemma. $\pi_{1}(M, A)$ contains no element of finite order.

Proof. The representation $\rho: \pi_{1}(M) \rightarrow \operatorname{Diff}^{+}\left(\mathbf{R}^{2}\right)$ defines a faithful representation of $\pi_{1}(M, A)$ in $\operatorname{Diff}^{+}\left(\mathbf{R}^{2}\right)$, each nontrivial element determining a fixed point free diffeomorphism of $\mathbf{R}^{2}$. If $\pi_{1}(M, A)$ contains an element of finite order, then there is $\alpha \in \pi_{1}(M, A)$ with prime order $p$. $\alpha$ generates a cyclic subgroup $\mathbf{Z}_{p} \subset \operatorname{Diff}^{+}\left(\mathbf{R}^{2}\right)$ acting freely on $\mathbf{R}^{2}$, so the orbit space $W$ of this action is a 2manifold with $\mathbf{R}^{2}$ as universal cover. $W$ must be an Eilenberg-Mac Lane space $K\left(\mathbf{Z}_{p}, 1\right)$ and so, by the standard homology theory of Eilenberg-Mac Lane spaces, $H_{q}\left(W ; \mathbf{Z}_{p}\right) \cong \mathbf{Z}_{p}, \forall q \geq 0$. This, however, contradicts the fact that $W$ is a 2 manifold. Q.E.D.

(7.6) Lemma. $\pi_{1}(M, A)$ is not cyclic.

Proof. If $\pi_{1}(M, A)$ is cyclic, then (7.4) and (7.5) imply $\pi_{1}(M, A)=\mathbf{Z}$. Let $Z \in V$ and consider the foliation $\mathscr{F}_{Z}$. We have two cases according to whether $\mathscr{F}_{Z}$ admits a compact leaf or not.

Suppose that $L$ is a compact leaf of $\mathscr{F}_{Z}$. By $(5.5), \pi_{1}(L, A)$ is free abelian of finite rank $\geq 1$. Since $\pi_{1}(L, A) \subset \pi_{1}(M, A)=\mathbf{Z}$, we conclude that $\pi_{1}(L, A)=\mathbf{Z}$. Then, again by (5.5), $L$ is fibered over $S^{1}$ with $A$ as fiber; hence $A$ is compact. By (4.4) 
it follows that $M$ is fibered over a compact parallelizable 2-manifold, hence over $T^{2}$, with $A$ as fiber. The exact homotopy sequence

$$
0 \rightarrow \pi_{1}(A) \rightarrow \pi_{1}(M) \rightarrow \pi_{1}\left(T^{2}\right) \rightarrow \pi_{0}(A)=0
$$

then shows $\pi_{1}(M, A) \cong \pi_{1}\left(T^{2}\right)=\mathbf{Z} \oplus \mathbf{Z}$, a contradiction.

Suppose, then, that $\mathscr{F}_{Z}$ admits no compact leaf. Then no leaf $L$ is closed, so in a neighborhood of $x \in \bar{L} \backslash L$ a transversal curve can be constructed which starts and ends on $L$. As usual, this shows the existence of a closed transversal $s$ to $\mathscr{F}_{Z}$. By (6.1) together with [12, Theorem 6.1], the lift of $s$ to $\hat{s}$ on $\hat{M}$ cannot be closed. Indeed, $\hat{s}$ cannot even begin and end on the same leaf of $\hat{\mathscr{F}}_{Z}$ (nor, a fortiori, on the same leaf of $\hat{\mathscr{F}}$ ) since this would imply the existence of a closed nullhomotopic transversal to $\hat{\mathscr{F}}_{Z}$ on $\hat{M}$, hence of such a transversal to $\mathscr{F}_{Z}$ on $M$, again contradicting (6.1) and [12, Theorem 6.1]. Thus $s$ represents a nontrivial element $\alpha \in \pi_{1}(M, A)$. Furthermore, $\rho(\alpha) \in \operatorname{Diff}^{+}\left(\mathbf{R}^{2}\right)$ cannot leave invariant any integral curve to $\tilde{Z}=p_{*}(\hat{Z})$ (where $p: \hat{M} \rightarrow \mathbf{R}^{2}$ is the projection defined by $\hat{M} \cong \hat{A} \times \mathbf{R}^{2}$ and $\tilde{Z}$ is well defined since $\hat{Z}$ is parallel along leaves of $\hat{\mathscr{F}}$ ). Since $\pi_{1}(M, A)=\mathbf{Z}$, the generator also fails to leave invariant any integral curve to $\tilde{Z}$, hence $0=\pi_{1}(L, A) \subset \pi_{1}(M, A)$ for any leaf $L$ of $\mathscr{F}_{Z}$, so $L \cong A \times \mathbf{R}$. It follows that any loop on $L$ is homotopic in $L$ to a loop on $A$, hence cannot be a limit cycle (since $A$ is a leaf of an $e$-foliation). By (5.4) and (5.5), $\hat{M}=\hat{L} \times \mathbf{R}$. But $0 \rightarrow \pi_{1}(A) \rightarrow \pi_{1}(L) \rightarrow 0$ is exact by the above, so $\pi_{1}(M, L) \cong \pi_{1}(M, A) \cong \mathbf{Z}$, hence by (5.5) $M$ is a fiber bundle over $S^{1}$ with $L$ as fiber. This contradicts the assumption that $L$ is noncompact.

Thus, in all cases we have shown that $\pi_{1}(M, A)$ cannot be cyclic. Q.E.D.

(7.7) Proposition. If $\pi_{1}(M, A)$ is abelian, then it is free abelian of finite rank $\geq 2$.

Proof. Since $M$ is compact, $\pi_{1}(M)$ is finitely generated. Thus $\pi_{1}(M, A)$ $=\pi_{1}(M) / \pi_{1}(A)$ is also finitely generated. By (7.5) this group must be free abelian (of finite rank), by (7.4) it is nontrivial; hence by (7.6) the rank of $\pi_{1}(M, A)$ is $\geq$ 2. Q.E.D.

8. Proof of the Main Theorem completed. Throughout this section we assume that $\pi_{1}(M, A)$ is abelian, hence that $\pi_{1}(M, A)=\mathbf{Z}^{r}, r \geq 2$. Our principal aim will be to recoordinatize $\mathbf{R}^{2}$ continuously so that the action of $\pi_{1}(M, A)$ will represent that group as a group of translations in $\mathbf{R}^{2}$. The final assertions of the Main Theorem will then follow readily.

The following is proven in [11, Théorème 2].

(8.1) Theorem (Moussu and Roussarie). Let $\mathscr{F}$ be a smooth transversally orientable foliation of codimension one. Suppose that $\mathscr{F}$ admits no vanishing cycles and that the image of $\pi_{1}(L) \rightarrow \pi_{1}(M)$ contains the commutator subgroup of $\pi_{1}(M)$, $\forall$ leaf $L$ of $\mathscr{F}$. If $\mathscr{F}$ has no compact leaf, then $\mathscr{F}$ admits no limit cycles (i.e., the holonomy of every leaf is trivial). 
This theorem, together with (6.1), gives the following.

(8.2) Corollary. If $\pi_{1}(M, A)$ is abelian and $\mathscr{F}_{Z}$ has no compact leaf, some $Z \in V-\{0\}$, then $\mathscr{F}_{Z}$ admits no limit cycles.

(8.3) Corollary. Let $Y \in V-\{0\}$ and suppose $\pi_{1}(M, A)$ abelian. If either all leaves or no leaves of $\mathscr{F}_{Y}$ are compact, there is a $C^{0}$ flow $\varphi: \mathbf{R} \times M \rightarrow M$ under which $\mathscr{F}_{Y}$ is invariant. Indeed, given $X \in V-\{0\}$ linearly independent of $Y, \varphi$ can so be chosen that the lines of flow are continuous reparametrizations of the integral curves to $X$.

Proof. If no leaves are compact, $\mathscr{F}_{Y}$ has no limit cycles by (8.2). If all are compact, then the holonomy of each leaf is finite and transverse orientablity implies no limit cycles. By [16, Theorem 6] the orthogonal trajectories to $\mathscr{F}_{Y}$ relative to any Riemannian metric on $M$ can be reparametrized so as to be the lines of flow for the desired $\varphi$. Actually, [16] supposes one of these trajectories to be a circle, but the fact that this circle is a trajectory for the metric in question is not really used. In particular, given $X \in V$ linearly independent of $Y$, a metric can be defined on $M$ such that $X$ is the unit normal to the leaves of $\mathscr{F}_{Y}$ and the corresponding $\varphi$ is as desired. Q.E.D.

(8.4) Lemma. The e-structure $\left(Y_{1}, Y_{2}\right)$ of $\mathscr{F}$ can so be chosen that both $\mathscr{F}_{Y_{1}}$ and $\mathscr{F}_{Y_{2}}$ verify the hypotheses of (8.3).

Proof. We consider two cases.

Case 1. Some leaf of $\mathscr{F}$ is compact By (4.4) every leaf is compact and these leaves are the fibers of a bundle $\pi: M \rightarrow T^{2}$. Let $X_{i}=\partial / \partial \theta_{i}, i=1,2$, be the basic fields on $T^{2}$ corresponding to the usual coordinates. Then the unique normal fields $Y_{i}^{\prime}$ such that $\pi_{*}\left(Y_{i}^{\prime}\right)=X_{i}, i=1,2$, give an $e$-structure in which all leaves of $\mathscr{F}_{Y_{i}^{\prime}}$ are compact.

Case 2. No leaf of $\mathscr{F}$ is compact. If the same is true of $\mathscr{F}_{Y_{i}}, i=1,2$, we are done. Otherwise, suppose some leaf $L \in \mathscr{F}_{Y_{1}}$ is compact. Let $A$ be a leaf of $\mathscr{F}$ with $A \subset L$. Since $\mathscr{F} \mid L$ is an $e$-foliation of codimension one and $A$ is not closed in $L$, (5.1) says that $A$ is everywhere dense in $L$. Since $L$ is compact, $L=\bar{A}=$ closure of $A$ in $M$. If $A^{\prime}$ is any other leaf of $\mathscr{F}$, then a sequence of flows corresponding to fields $X_{j} \in V$ provide a diffeomorphism $\varphi: M \rightarrow M$ leaving $\mathscr{F}$ invariant with $\varphi(A)=A^{\prime}$. Thus the closure of each leaf $A$ of $\mathscr{F}$ is a smooth codimension one submanifold $\bar{A}$ of $M$ which is a union of leaves of $\mathscr{F}, \mathscr{F} \mid \bar{A}$ being an $e$-foliation. If two of these submanifolds intersect, some $A$ belongs to both, hence both coincide with $\bar{A}$. Applying the flow $\psi_{t}$ generated by $Y_{2}$ to $L$, we see that $\psi_{t}(L)$ is one of these manifolds, $\forall t$, and since $Y_{2} \mid L$ is transverse to $L$, a normal neighborhood of $L$ is smoothly foliated by these manifolds. Again moving this neighborhood about by a sequence of flows generated by fields $X_{j} \in V$, we see that $M$ is smoothly foliated by the leaves $\bar{A}$. Call this foliation $\overline{\mathscr{F}}$. Clearly $\overline{\mathscr{F}}$ is without holonomy, hence is transversally orientable. Choose a tranverse orientation. 
The original $e$-structure of $\mathscr{F}$ defines a bundle-like metric. Using this metric, define a normal field $Y_{1}^{\prime}$ to $\mathscr{F}$ by requiring $Y_{1}^{\prime} \perp$ leaves of $\mathscr{F}, Y_{1}^{\prime}$ tangent to the leaves of $\overline{\mathscr{F}},\left\|Y_{1}^{\prime}\right\| \equiv 1$, and, if $X \in \mathcal{X}(M)$ is transverse to $\overline{\mathscr{F}}$ and positively oriented, $\left(Y_{1}^{\prime}, X\right)$ should determine the same transverse orientation of $\mathscr{F}$ as $\left(Y_{1}, Y_{2}\right)$.

If $U$ is a local product neighborhood for $\mathscr{F}, f: U \rightarrow \mathbf{R}^{2}$ a distinguished map (cf. $[4,1.2])$, the local leaves of $\overline{\mathscr{F}} \mid U$ are carried by $f$ to smooth curves in $\mathbf{R}^{2}$ and $f_{* x}\left(Y_{1 x}^{\prime}\right)$ is tangent to the curve through $f(x), \forall x \in U$. If $f(x)=f(y)$, then $f_{* x}\left(Y_{1 x}^{\prime}\right)$ and $f_{* y}\left(Y_{1 y}^{\prime}\right)$ are positive multiples of each other. Since $\left\|Y_{1 x}^{\prime}\right\|=1$ $=\left\|Y_{1 y}^{\prime}\right\|$ in a bundle-like metric, we have $f_{* x}\left(Y_{1 x}^{\prime}\right)=f_{* y}\left(Y_{1 y}^{\prime}\right)$. Thus $f_{*}\left(Y_{1}^{\prime}\right)$ is a well-defined field and $Y_{1}^{\prime}$ is everywhere parallel along leaves of $\mathscr{F}$. Define $Y_{2}^{\prime}$ normal to $\mathscr{F}$ by requiring that $\left\|Y_{2}^{\prime}\right\| \equiv 1, Y_{2}^{\prime} \perp Y_{1}^{\prime}$ everywhere, and that $\left(Y_{1}^{\prime}, Y_{2}^{\prime}\right)$ determines the same transversal orientation as $\left(Y_{1}, Y_{2}\right)$. Then $\left(Y_{1}^{\prime}, Y_{2}^{\prime}\right)$ is an $e$ structure for $\mathscr{F}$.

$\mathscr{F}_{Y_{1}^{\prime}}=\overline{\mathscr{F}}$ and has all its leaves compact. If $L^{\prime} \in \mathscr{F}_{Y_{2}^{\prime}}, L \in \mathscr{F}_{Y^{\prime}}$, then $L$ is transversal to $L^{\prime}$, so $L \cap L^{\prime}$ is an at most countable union of leaves of $\mathscr{F}$. Since the connected components of $L \cap L^{\prime}$ are not compact, although $L$ is compact, we see that no $L^{\prime}$ can be compact. Q.E.D.

Combining (8.4) and (8.3), we obtain the following.

(8.5) Corollary. Let $\left(Y_{1}, Y_{2}\right)$ be an e-structure for $\mathcal{F}$ as in (8.4). Then there are $C^{0}$ flows $\varphi$ and $\psi$ on $M$ such that $\varphi$ leaves invariant each leaf of $\mathscr{F}_{Y_{1}}$ and permutes the leaves of $\mathscr{F}_{Y_{2}}$, while $\psi$ leaves invariant each leaf of $\mathscr{F}_{Y_{2}}$ and permutes those of $\mathscr{F}_{Y_{1}}$. Thus $\varphi$ and $\psi$ also permute the leaves of $\mathscr{F}$.

On $\hat{M}$ let $\hat{\varphi}, \hat{\psi}$ be the flows obtained by lifting $\varphi$ and $\psi$ of $(8.5)$. $\hat{\varphi}$ and $\hat{\psi}$ permute the leaves of $\hat{\mathscr{F}}$; hence they define $C^{0}$ flows $\tilde{\varphi}$ and $\tilde{\psi}$ on $\mathbf{R}^{2}$.

(8.6) Lemma. Each flow line of $\tilde{\varphi}$ intersects each flow line of $\tilde{\psi}$ in exactly one point. Furthermore, $\tilde{\varphi}_{t} \circ \tilde{\psi}_{s}=\tilde{\psi}_{s} \circ \tilde{\varphi}_{t}, \forall t, s \in \mathbf{R}$. Consequently, $\varphi_{t} \psi_{s}(A)=\psi_{s} \varphi_{t}(A), \forall$ leaf $A$ of $\mathscr{F}$.

Proof. Observe that each leaf of $\hat{\mathscr{F}}_{\hat{p}_{1}}$ projects (under the canonical map $\hat{A} \times \mathbf{R}^{2} \rightarrow \mathbf{R}^{2}$ ) onto the trajectory of a flow line of $\tilde{\varphi}$ and similarly for $\hat{\mathscr{F}}_{P_{2}}$ and $\tilde{\psi}$. Since $\mathscr{F}_{Y_{2}}$ admits no limit cycles, it follows as in [15, pp. 171-172] that each $\hat{\varphi}$ flow line meets each $L \in \hat{\mathscr{F}}_{\hat{P}_{2}}$ once and only once. The assertion about the flow lines of $\tilde{\varphi}$ and $\tilde{\psi}$ follows immediately.

From these considerations it also follows that each leaf $L$ of $\tilde{\mathscr{F}}_{\hat{P}_{1}}$ intersects each

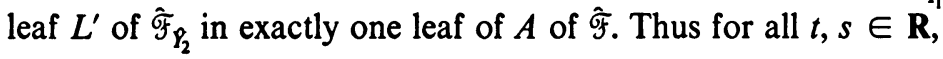

$$
\begin{aligned}
\hat{\varphi}_{t} \hat{\psi}_{s}(A) & =\hat{\varphi}_{t} \hat{\psi}_{s}\left(L \cap L^{\prime}\right)=\hat{\varphi}_{t}\left(L^{\prime}\right) \cap \hat{\psi}_{s}(L) \\
& =\hat{\psi}_{s} \hat{\varphi}_{t}\left(L \cap L^{\prime}\right)=\hat{\psi}_{s} \hat{\varphi}_{t}(A) .
\end{aligned}
$$

It follows that $\tilde{\varphi}_{t} \circ \tilde{\psi}_{s}=\tilde{\psi}_{s} \circ \tilde{\varphi}_{t}$. Q.E.D. 
Thus we obtain a continuous faithful simply transitive action of the topological group $\mathbf{R}^{2}$ on the topological space $\mathbf{R}^{2}$. Applying the group element $(t, s)$ to the origin $(0,0)$ defines a homeomorphism $\theta: \mathbf{R}^{2} \rightarrow \mathbf{R}^{2}, \theta(t, s)=\tilde{\varphi}_{t} \tilde{\psi}_{s}(0,0)=$ $\tilde{\psi}_{s} \tilde{\varphi}_{t}(0,0)$. We interpret $\theta^{-1}$ as a continuous reparametrization of $R^{2}$. Under this reparametrization, the action of $\pi_{1}(M, A)$ on $\mathbf{R}^{2}$ takes a pleasantly simple form.

(8.7) Proposition. Let $\alpha \in \pi_{1}(M, A)$ and set $(a, b)=\theta^{-1} \alpha \theta(0,0)$. Then $\theta^{-1} \alpha \theta: \mathbf{R}^{2}$ $\rightarrow \mathbf{R}^{2}$ is translation by the vector $(a, b)$.

Proof. Remark that $\alpha$ commutes with the flows $\tilde{\varphi}, \tilde{\psi}$ since $\hat{\varphi}, \hat{\psi}$ are lifted from M. Also, $\alpha(0,0)=\alpha \theta(0,0)=\theta(a, b)=\tilde{\varphi}_{a} \tilde{\psi}_{b}(0,0)$. Thus, for all $(t, s) \in \mathbf{R}^{2}$, $\alpha \theta(t, s)=\alpha \tilde{\varphi}_{t} \tilde{\psi}_{s}(0,0)=\tilde{\varphi}_{t} \tilde{\psi}_{s} \alpha(0,0)=\tilde{\varphi}_{t} \tilde{\psi}_{s} \tilde{\varphi}_{a} \tilde{\psi}_{b}(0,0)=\tilde{\varphi}_{t+a} \tilde{\psi}_{s+b}(0,0)=$ $\theta(t+a, s+b)$, so $\theta^{-1} \alpha \theta(t, s)=(t+a, s+b)$. Q.E.D.

We can now complete the proof of the Main Theorem. Remark first that the covering space $\tilde{M} \rightarrow M$ obtained from $\hat{M}$ by dividing out the action of $\pi_{1}(A)$ has the form $\tilde{M} \cong A \times \mathbf{R}^{2}$ with $\pi_{1}(M, A)$ as the group of deck transformations. Since $\pi_{1}(M, A)=\mathbf{Z}^{r}$ is also represented as a group of translations of $\mathbf{R}^{2}$, it sits as an additive subgroup $\mathbf{Z}^{r} \subset \mathbf{R}^{2}, r \geq 2$. Let $G \subset \mathbf{R}^{2}$ be the closure of this subgroup; hence $G$ is a Lie subgroup of $\mathbf{R}^{2}$ and we consider three cases.

For case 1 , let $\operatorname{dim}(G)=0$. Then $r=2$ and $G=\mathbf{Z}^{2}$ is a lattice group in $\mathbf{R}^{2}$, so each leaf of $\mathscr{F}$ is closed in $M$. By (4.4), $\mathscr{F}$ must foliate $M$ by the fibers of a smooth bundle $M \rightarrow T^{2}$.

For case 2 , take $\operatorname{dim}(G)=1$. Then $G$ is a discrete family of parallel lines. It follows that $M$ has a $C^{0}$ foliation of codimension one with leaves the closure of the leaves of $\mathscr{F}$. Indeed, at least one of the flows $\varphi$ or $\psi$ of (8.5), say $\varphi$, has the property that for any $A \in \mathscr{F}$ and some $\varepsilon>0$, the map $h: \bar{A} \times(-\varepsilon, \varepsilon) \rightarrow M$ defined by $h(x, t)=\varphi_{t}(x)$ is a homeomorphism onto an open neighborhood of $\bar{A}$. Thus $M$ is fibered by the leaves $\bar{A}$ over a compact connected one dimensional manifold. This is the desired topological bundle $M \rightarrow S^{1}$.

The third case has $\operatorname{dim}(G)=2$. Thus $\mathbf{Z}^{r}$ is everywhere dense in $\mathbf{R}^{2}$, so each $A \in \mathscr{F}$ is everywhere dense in $M$. This completes the proof of the Main Theorem.

9. Proofs of the corollaries. We consider Corollaries A through F as formulated in the introduction.

The Main Theorem together with (3.1) and the remark immediately following (3.1) give Corollary A for codimension two. Indeed, $M$ compact with $\pi_{1}(M)$ finite implies $\hat{M}$ compact. If $\mathscr{F}$ is transversally almost parallelizable, then $\hat{\mathscr{F}}$ is transversally parallelizable and the Main Theorem is contradicted. Similarly, in codimension one (5.5) is contradicted.

We prove Corollary B. Here $M$ is a compact connected $n$-manifold, $n \geq 5$, and $\pi_{k}(M)=0,2 \leq k \leq n-3$. If $\mathscr{F}$ is a codimension two $e$-foliation of $M$, then $\hat{M} \cong \hat{A} \times \mathbf{R}^{2}$ and

$$
\pi_{k}(A)=\pi_{k}(\hat{A})=\pi_{k}(\hat{M})=\pi_{k}(M)=0
$$


for $2 \leq k \leq n-3$. If $\pi_{n-2}(\hat{A})=0$, then, by the Hurewicz theorem [7], $H_{n-2}(\hat{A})$ $=0$. Since $\hat{A}$ is a simply connected $(n-2)$-manifold, it has vanishing homology in all positive dimensions; hence $\pi_{k}(\hat{A})=0, \forall k \geq 0$, by Hurewicz. $\hat{A}$ is a CWcomplex; hence by a theorem of J. H. C. Whitehead [8, p.125], $\hat{A}$ is contractible. Thus $\hat{M}$ is contractible. If, on the other hand, $\pi_{n-2}(\hat{A}) \neq 0$, the same holds for $H_{n-2}(\hat{A})$, so $\hat{A}$ is a compact simply connected integral homology sphere. In particular, $\pi_{n-2}(\hat{A})=\mathbf{Z}$ and the generator

$$
f: S^{n-2} \rightarrow \hat{A}
$$

is a homotopy equivalence. Furthermore, $A=\pi(\hat{A})$ is compact; hence our standard application of (4.4) shows that the foliation of $M$ is a fiber bundle over $T^{2}$. Corollary B is completely proven.

The proof of Corollary $\mathbf{C}$ is simply a refinement of the proof of Corollary B. Here $\hat{A}$ is a contractible 2-manifold if $\pi_{2}(\hat{A})=0$; hence $\hat{A} \cong \mathbf{R}^{2}$ and $\hat{M} \cong \mathbf{R}^{4}$. If $\pi_{2}(\hat{A}) \neq 0$, then $\hat{A}$ is a homotopy 2 -sphere, hence $\hat{A}=S^{2}$. The only 2 manifolds $A$ with $S^{2}$ as universal cover are $S^{2}$ and $P^{2}$, so Corollary $\mathrm{C}$ is proven.

Corollary $\mathrm{D}$ is completely trivial. $\hat{A}$ is a simply connected 1-manifold; hence $\hat{A} \cong \mathbf{R}$ and $\hat{M} \cong \mathbf{R}^{3}$.

For Corollary $\mathrm{E}$ we have the hypothesis that $\pi_{1}(M)$ is abelian; hence $\pi_{1}(M, A)=\mathbf{Z}^{k}, k \geq 2$. Dividing out the action of $\pi_{1}(A)$ on $\hat{M}$ yields the covering space $\tilde{M}=A \times \mathbf{R}^{2} \rightarrow M$ with $\mathbf{Z}^{k}=\pi_{1}(M, A)$ as the group of covering transformations. Consider this group as acting from the right and also consider its standard (left) action on $\mathbf{R}^{k}$ as the group of covering transformations over $T^{k}$. Form the twisted product

$$
X=\tilde{M} \times_{\mathbf{Z}^{k}} \mathbf{R}^{k}
$$

This is the total space of two fiber bundles

$$
\mathbf{R}^{k} \rightarrow X \underset{q}{\rightarrow} M, \quad \tilde{M} \rightarrow X \underset{p}{\rightarrow} T^{k},
$$

where, for definiteness, we take $i(y)=[y, 0], \forall y \in \tilde{M}$. The first of these fibrations shows that $q$ is a homotopy equivalence, so we let $r: M \rightarrow X$ be a homotopy inverse. Also let $j: A \rightarrow \tilde{M}=A \times \mathbf{R}^{2}$ be the homotopy equivalence defined by $j(a)=(a, 0), \forall a \in A$. Let $f: A \rightarrow M$ be the leaf inclusion covered by $j$. The diagram

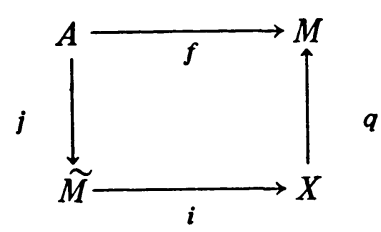


is commutative; hence

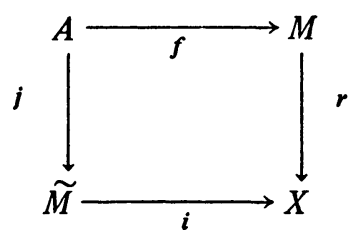

is homotopy commutative. Defining $t: M \rightarrow T^{k}$ by $t=p \circ r$ gives a homotopy commutative diagram

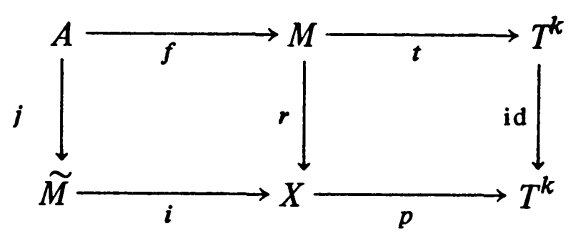

in which the vertical arrows are homotopy equivalences and the bottom row is a fiber bundle. Since $f$ is the immersion of a leaf, the proof of Corollary $\mathrm{E}$ is complete.

It remains to prove Corollary $\mathrm{F}$. Under the hypotheses of that corollary, $\pi_{1}(A)=\mathbf{Z}^{r}, 0 \leq r \leq n-2, \pi_{1}(M, A)=\mathbf{Z}^{k}, k \geq 2$, and, $\pi_{1}(M)$ being abelian, the exact sequence

$$
0 \rightarrow \pi_{1}(A) \rightarrow \pi_{1}(M) \rightarrow \pi_{1}(M, A) \rightarrow 0
$$

shows that $\pi_{1}(M)=\mathbf{Z}^{m}, m=r+k$. Since $\hat{A}=\mathbf{R}^{n-2}$, the Main Theorem shows that $\hat{M}=\mathbf{R}^{n}$, so $M$ is an Eilenberg-Mac Lane space $K\left(\mathbf{Z}^{m}, 1\right)$. Since $T^{m}$ is also a $K\left(\mathbf{Z}^{m}, 1\right)$, we see that $M$ has the homotopy type of $T^{m}$. Since these are both compact manifolds of respective dimensions $n$ and $m$, it follows that $m=n$ and Corollary $\mathrm{F}$ is proved.

10. Strong transverse $e$-structures. In this section we assume some familiarity with the Haefliger cocycles associated to a codimension $q$ foliation [5], [2, p. 40]. Recall that these consist of an open cover $\left\{U_{\alpha}\right\}$ of $M$, submersions $f_{\alpha}: U_{\alpha} \rightarrow \mathbf{R}^{q}$, and, for each $x \in U_{\alpha} \cap U_{\beta}$, the germ $g_{\alpha \beta}^{x}$ of a diffeomorphism of a neighborhood of $f_{\alpha}(x)$ onto a neighborhood of $f_{\beta}(x)$. The germs $f_{\alpha}^{x}$ and $f_{\beta}^{x}$ are related by

$$
f_{\beta}^{x}=g_{\alpha \beta}^{x} \circ f_{\alpha}^{x} .
$$

The tangent spaces to $\mathbf{R}^{q}$ all identify canonically with $\mathbf{R}^{q}$, hence $d g_{\alpha \beta}^{x} \in G l_{q}$ is well defined.

(10.1) Definition. Let $H \subset G l_{q}$ be a Lie subgroup. Let $\mathscr{F}$ be a codimension $q$ foliation of $M$. A strong transverse $H$-structure for $\mathscr{F}$ is a Haefliger cocycle $\left\{U_{\alpha}, f_{\alpha}, g_{\alpha \beta}^{x}\right\}$ for $\mathscr{F}$ such that $d g_{\alpha \beta}^{x} \in H, \forall \alpha, \beta$ and $\forall x \in U_{\alpha} \cap U_{\beta}$. F together with a choice of such a cocycle is called a strong $H$-foliation. 
Since $\gamma_{\alpha \beta}: U_{\alpha} \cap U_{\beta} \rightarrow G l_{q}$ defined by $\gamma_{\alpha \beta}(x)=d g_{\alpha \beta}^{x}$ is constant along local leaves and gives a system of transition functions for the normal bundle $Q$, the next proposition is elementary.

(10.2) Proposition. A strong transverse $H$-structure for $\mathscr{F}$ implies the existence of a transverse $H$-structure for $\mathscr{F}$.

The converse of (10.2) is generally false, as we shall see.

Let $\left\{U_{\alpha}, f_{\alpha}, g_{\alpha \beta}^{x}\right\}$ be a strong transverse $e$-structure for a codimension $q$ foliation F, $\left(Y_{1}, \ldots, Y_{q}\right)$ the corresponding transverse $e$-structure such that all $f_{\alpha^{*}}\left(Y_{i} \mid U_{\alpha}\right)$ $=\partial / \partial x_{i}$ on $f_{\alpha}\left(U_{\alpha}\right), i=1, \ldots, q$. As usual, let $V$ be the span of the $Y_{i}$ s over $\mathbf{R}$.

(10.3) Lemma. For $\mathscr{F}$ as above and $0 \neq Z \in V$, $\mathscr{F}_{Z}$ is a strong e-foliation.

Proof. Since $\left[\partial / \partial x_{i}, \partial / \partial x_{j}\right] \equiv 0$ in $\mathbf{R}^{q}$, our above definitions imply that $\left[Y_{i}, Y_{j}\right] \in \Gamma(E)(E$ the tangent bundle to g) $, \forall i, j$; hence that $[Z, W] \in \Gamma(E)$, $\forall Z, W \in V$. Let $V^{\prime}=\{W \in V: W \perp Z\}$. Then by (1.5) it follows that each $W \in V^{\prime}$ is parallel along all the leaves of $\mathscr{F}_{Z}$. Furthermore, a choice of basis $W_{1}, \ldots, W_{q-1}$ in $V^{\prime}$ and a once and for all linear recoordinatization of $\mathbf{R}^{q}$ with coordinates $y_{1}, \ldots, y_{q}$ gives

$$
\begin{aligned}
f_{\alpha^{*}}\left(W_{i} \mid U_{\alpha}\right) & =\partial / \partial y_{i}, \quad i=1, \ldots, q-1, \\
f_{\alpha^{*}}\left(Z \mid U_{\alpha}\right) & =\partial / \partial y_{q},
\end{aligned}
$$

for all $\alpha$. Thus, if $p: \mathbf{R}^{q} \rightarrow \mathbf{R}^{q-1}$ is projection along the $y_{q}$-axis, the maps $p \circ f_{\alpha}=\bar{f}_{\alpha}: U_{\alpha} \rightarrow \mathbf{R}^{q-1}$ are submersions defining $\mathscr{F}_{Z}$ and related by $\bar{g}_{\alpha \beta}^{x}$ such that $d \bar{g}_{\alpha \beta}^{x}=$ identity. Q.E.D.

(10.4) Theorem. Let $M$ be a compact connected manifold with a strong e-foliation $\mathcal{F}$ of codimension $q$. Then the universal cover $\hat{M} \cong \hat{A} \times \mathbf{R}^{q}$ where $\hat{A}$ is the universal cover of the typical leaf $A$ of $\mathscr{F}$. The homomorphism $\pi_{1}(A) \rightarrow \pi_{1}(M)$ is a monomorphism onto a normal subgroup of $\pi_{1}(M)$ and the group $\pi_{1}(M, A)=\pi_{1}(M) / \pi_{1}(A)$ is free abelian of finite rank $\geq q$. If the rank is $q$, then the foliation is a fiber bundle over $T^{q}$.

Proof. $\mathscr{F}_{1}=\mathscr{F}_{Y_{1}}$ is a strong $e$-foliation by (10.3); hence we can form the strong $e$-foliation $\mathscr{F}_{2}=\left(\mathscr{F}_{Y_{1}}\right)_{Y_{2}}$. Proceeding in this way, we obtain a sequence $\mathscr{F}=\mathscr{F}_{0}, \mathscr{F}_{1}$, $\mathscr{F}_{2}, \ldots, \mathscr{F}_{q-1}$ of strong $e$-foliations, $\operatorname{codim}\left(\mathscr{F}_{i}\right)=q-i$, each $\mathscr{F}_{i}(i \geq 1)$ having its leaves $e$-foliated by leaves of $\mathscr{F}_{i-1}$. Each of these $e$-foliations of a leaf of $\mathscr{F}_{i}$ is transversally complete by the compactness of $M$. Thus, repeated application of (5.5) gives $\hat{M} \cong \hat{A} \times \mathbf{R}^{q}$ as desired. As usual, this implies $\pi_{1}(A) \rightarrow \pi_{1}(M)$ oneone. If $p: \hat{M} \rightarrow \mathbf{R}^{q}$ is the corresponding projection, the fields $\tilde{Y}_{i}=p_{*}\left(\hat{Y}_{i}\right)$ are complete and all $\left[\tilde{Y}_{i}, \tilde{Y}_{j}\right] \equiv 0$, so $\mathbf{R}^{q}$ can so be coordinatized that $\tilde{Y}_{i}=\partial / \partial y_{i}, i$ $=1, \ldots, q$. Thus, the image of the natural homomorphism $\rho: \pi_{1}(M)$ $\rightarrow \operatorname{Diff}^{+}\left(\mathbf{R}^{q}\right)$ consists of diffeomorphisms leaving all $\partial / \partial y_{i}$ invariant, hence of 
translations in $\mathbf{R}^{q}$. Thus $\rho\left(\pi_{1}(M)\right)$ is free abelian, finitely generated since $M$ is compact, and $\rho(\alpha)$ has a fixed point in $\mathbf{R}^{q}$ only if $\rho(\alpha)=$ identity. Thus $\operatorname{Ker}(\rho)=\pi_{1}(A)$ and $\pi_{1}(M, A)$ is free abelian of finite rank. If the rank were $<q$ the translation vectors for elements of $\operatorname{Im}(\rho)$ would span a proper subspace of $\mathbf{R}^{q}$ and it would follow that $M$ could not be compact. Similarly, if the rank were $q$ but $\pi_{1}(M, A)$ did not act as a full $q$-dimensional lattice group on $\mathbf{R}^{q}, M$ could not be compact. Thus, if the covering space $A \times \mathbf{R}^{q} \rightarrow M$ has covering transformations $\pi_{1}(M, A)=\mathbf{Z}^{q}$, the foliation $\mathscr{F}$ will be a fiber bundle over $T^{q}$. Q.E.D.

As remarked in the introduction, the foliation of $S^{1} \times S^{3}$ by leaves $S^{1} \times \mathrm{pt}$. is an $e$-foliation not satisfying the conclusion of (10.4). Thus the converse of (10.2) is false.

Since $T^{q}$ admits a global frame field $\left(X_{1}, \ldots, X_{q}\right)$ with all $\left[X_{i}, X_{j}\right] \equiv 0$, it is immediate that any fibration $M \rightarrow T^{q}$ is a strong $e$-foliation. Since $T^{2}$ is the only compact connected parallelizable 2-manifold, (4.4) gives the following result.

(10.5) Proposition. If $\mathscr{F}$ is a codimension two e-foliation of compact $M$, and if $\mathscr{F}$ admits a closed leaf, then $\mathscr{F}$ admits a strong transversal e-structure.

The general philosophy of this subject seems to be that the leaf space of an $e$ foliation is "trying to be" a parallelizable manifold, while that of a strong $e$ foliation is trying to admit the additional structure of a flat Riemannian manifold. Since all parallelizable 2-manifolds admit flat Riemannian metrics, the following conjecture, supported by (10.5), seems reasonable.

(10.6) Conjecture. Any codimension two e-foliation of compact $M$ admits a strong transversal e-structure.

Comparing (10.4) with the Main Theorem we find that the possibility that $\pi_{1}(M, A)$ is not abelian is an obstruction to (10.6). We do not know whether this ever happens. Noticing that a strong $e$-structure $\left\{U_{\alpha}, f_{\alpha}, \gamma_{\alpha \beta}^{x}\right\}$ is precisely a cocycle for which every $\gamma_{\alpha \beta}^{x}$ is the germ of a translation in $\mathbf{R}^{q}$, we offer the following partial verification of (10.6).

(10.7) Theorem. Let $\mathscr{F}$ be a codimension two e-foliation of compact connected $M$. If $\pi_{1}(M, A)$ is abelian, then there is a class $C^{0}$ Haefliger cocycle $\left\{U_{\alpha}, f_{\alpha}, \gamma_{\alpha \beta}^{x}\right\}$ for $\mathcal{F}^{\circ}$ such that each $\gamma_{\alpha \beta}^{x}$ is the germ of a translation in $\mathbf{R}^{\mathbf{2}}$.

Proof. Cover $\hat{M}=\hat{A} \times \mathbf{R}^{2}$ by product neighborhoods $V_{\alpha} \times W_{\alpha}, V_{\alpha}$ a coordinate neighborhood in $\hat{A}, W_{\alpha}$ open in $\mathbf{R}^{2}$, such that the covering map $p: \hat{M} \rightarrow M$ carries each $V_{\alpha} \times W_{\alpha}$ homeomorphically onto an open set $U_{\alpha} \subset M$. Let $p_{\alpha}=p \mid V_{\alpha} \times W_{\alpha}$. By (8.7), our willingness to sacrifice differentiability allows us to assume that the covering transformations $\gamma \in \pi_{1}(M)$ have the form $\gamma(x, t, s)=(\bar{\gamma}(x, t, s), t+a, s$ $+b$ ) for suitable continuous $\bar{\gamma}: \hat{M} \rightarrow \hat{A}$ and constants $a$ and $b$. It follows that $\left\{U_{\alpha}, f_{\alpha}\right\}$ with $f_{\alpha}\left(p_{\alpha}(x, t, s)\right)=(t, s)$ defines a $C^{0}$ cocycle $\left\{U_{\alpha}, f_{\alpha}, \gamma_{\alpha \beta}^{x}\right\}$ in which $\gamma_{\alpha \beta}^{x}$ is always the germ of a translation. Q.E.D. 


\section{REFERENCES}

1. L. V. Ahlfors and L. Sario, Riemann surfaces, Princeton Math. Series, no. 26, Princeton Univ. Press, Princeton, N. J., 1960. MR 22 \#5729.

2. R. Bott, Lectures on characteristic classes and foliations (notes by L. Conlon), Lecture Notes in Math., no. 279, Springer-Verlag, New York, 1972, 1-80.

3. C. Chevalley, Theory of Lie groups. Vol. I, Princeton Math. Series, vol. 8, Princeton Univ. Press, Princeton, N. J., 1946. MR 7, 412.

4. A. Haefliger, Variétés feuilletées, Ann. Scuola Norm. Sup. Pisa (3) 16 (1962), 367-397. MR 32 \#6487.

5._- Feuilletages sur les variétés ouvertes, Topology 9 (1970), 183-194. MR 41 \#7709.

6.- Homotopy and integrability, Manifolds-Amsterdam 1970 (Proc. Nuffic Summer School), Lecture Notes in Math., vol. 197, Springer, Berlin, 1971, pp. 133-163. MR 44 \#2251.

7. S. T. Hu, Homotopy theory, Pure and Appl. Math., vol. 8, Academic Press, New York, 1959. MR 21 \#5186.

8. A. L. Lundell and S. Weingram, The topology of CW complexes, Van Nostrand, New York, 1969.

9. R. Moussu, Feuilletages sans holonomie d'une variété fermée, C. R. Acad. Sci. Paris Sér. A-B 270 (1970), Al308-Al311. MR 41 \#7710.

10._, Sur un théorème de Novikov, Rev. Colombiana Mat. 3 (1969), 51-81. MR 41 \#2703.

11. R. Moussu and R. Roussarie, Une condition suffisante pour qu'un feuilletage soit sans holonomie, C. R. Acad. Sci. Paris Sér. A-B 271 (1970), A240-A243. MR 43 \#2732.

12. S. P. Novikov, Topology of foliations, Trudy Moskov. Mat. Obšč. 14 (1965), 248-278 = Trans. Moscow Math. Soc. 1965, 268-304. MR 34 \#824.

13. J. S. Pasternack, Foliations and compact Lie group actions, Comment. Math. Helv. (1972), 55-65.

14. B. L. Reinhart, Foliated manifolds with bundle-like matrics, Ann. of Math. (2) 69 (1959), 119-132. MR 21 \#6004.

15. H. Rosenberg, Actions of $\mathbf{R}^{n}$ on manifolds, Comment. Math. Helv. 41 (1966/67), 170-178. MR 34 \#6794.

16. R. Sacksteder, Foliations and pseudogroups, Amer. J. Math. 87 (1965), 79-102. MR 30 \#268.

17. P. Schweitzer, Counterexamples to the Seifert conjecture and opening closed leaves of foliatons, Ann. of Math. (to appear).

Department of Mathematics, St. Louis University, St. Louis, Missouri 63103

Department of Mathematics, Washington University, St. Louis, Missouri, 63130 (Current address) 